\title{
A STRONG CONVERGENCE THEOREM FOR STATIONARY GAUSSIAN SEQUENCES IN A HILBERT SPACE
}

\author{
CHANDRAKANT M. DEO
}

\begin{abstract}
An a.s. functional LIL is proved for stationary Gaussian sequences taking values in a Hilbert space.
\end{abstract}

1. Introduction. In [5] it was proved that a stationary real-valued Gaussian sequence obeys Strassen's LIL if the correlation coefficients go to zero fast enough. It was also shown there that if the limit set is to be the Strassen's set then the condition on the correlation sequence cannot be significantly relaxed. The object of this note is to extend this result to stationary Gaussian sequences taking values in a Hilbert space.

T. L. Lai [11] discovered other Strassen-type theorems for a real-valued Wiener process. More general normalizing constants were introduced by Csörgö and Révész in [4] and finally Chan, Csörgö and Révész [3] give a theorem which includes Strassen's and Lai's theorems as particular cases.

It is in this more inclusive form of [3] that we extend the result in [5] to $H$-valued sequences. The method of proof employed is based on the elegant work of Kuelbs [9] and Carmona and Kôno [2]. As a by-product of this method of proof we also quickly obtain Theorem 2 below which extends the results in Kuelbs and Lepage [10] and in [3].

2. Main results. $H$ is a real separable Hilbert space throughout with inner product $\langle$,$\rangle . Let X$ be an $H$-valued, zero-mean Gaussian random variable. Then the distribution of $x$ is completely determined by its covariance operator $K$ defined by

$$
\langle K u, v\rangle=E\{\langle u, X\rangle\langle v, X\rangle\}, \quad \forall u, v \in H .
$$

If $X, Y$ are two $H$-valued r.v's having joint (i.e. on $H \times H$ ) zero-mean, Gaussian distribution then the joint distribution of $(X, Y)$ is completely determined by the marginal covariance operators of $X, Y$ and their cross-covariance operator $K_{X, Y}$ defined by

$$
\left\langle K_{X, Y} u, v\right\rangle=E\{\langle u, X\rangle\langle v, Y\rangle\} \quad \forall u, v \in H .
$$

The covariance as well as the cross-covariance operators are trace-class operators. The covariance operators, in addition, are positive. These operators are discussed in Baker [1]. Facts about general trace-class operators (e.g. that they form a Banach space under the trace-norm) which we need can all be found in [7].

Received by the editors October 4, 1978 and, in revised form, May 14, 1979.

AMS (MOS) subject classifications (1970). Primary 60B05, 60F15. 
Let $C_{H}[0,1]$ be the Banach space of all continuous functions from the unit interval $[0,1]$ into $H$ equipped with the supremum norm. If $\mu$ is a zero-mean, Gaussian measure on $H$, there exist a $\mu$-Wiener process $\{W(t): 0<t<1\}$ with sample paths in $C_{H}[0,1]$ i.e. $W$ has independent increments with $W(0)=0$ and the probability law of $\left(t_{2}-t_{2}\right)^{-1 / 2}\left[W\left(t_{2}\right)-W\left(t_{1}\right)\right]$ is $\mu$ for all $0<t_{1}<t_{2}<1$. The Gaussian measure induced on $C_{H}[0,1]$ by $W$ is supported by the closure of its RKHS and let us denote by $U_{\mu}$ the compact subset of $C_{H}[0,1]$ which is the unit ball of this RKHS. A nicely detailed discussion of this is given in [10].

Now let $\left(X_{n}: n \geqslant 1\right)$ be a stationary sequence of zero-mean, $H$-valued, Gaussian random variables. Denote by $K_{0}$ the covariance operator of $X_{n}$ and by $K_{n}$ the cross-covariance operator of $X_{m}$ and $X_{m+n}$. We will assume that

$$
\text { trace-norm of } K_{n}=O\left(n^{-1-\beta}\right) \text { for some } \beta>0 \text {. }
$$

The covariance operator of $n^{-1 / 2} S_{n}=n^{-1 / 2} \sum_{i=1}^{n} X_{i}$ is given by $K_{0}+$ $\sum_{i=1}^{n-1}(1-i / n)\left(K_{i}+K_{i}^{*}\right)$ where $K_{i}^{*}$ is the transpose of $K_{i}$. Under our condition (1) this covariance operator of $n^{-1 / 2} S_{n}$ is easily seen to converge in the trace-norm topology to the covariance operator $L=K_{0}+\sum_{i=1}^{\infty}\left(K_{i}+K_{i}^{*}\right)$, the series here being convergent in the trace-norm topology. Let $\mu$ be the zero-mean Gaussian measure corresponding to the operator $L$ and $U_{\mu}$ be as defined above.

Now consider a sequence of integers $\left(a_{n}\right)$ satisfying (i) $a_{n}<n, a_{n} \uparrow \infty$ and (ii) $a_{n} / n$ is decreasing. Write $b_{n}=\left\{\log \left(n / a_{n}\right)+\log \log n\right\}$ and

$$
\begin{aligned}
g_{n}(t) & =\left(2 a_{n} b_{n}\right)^{-1 / 2}\left\{S_{n-a_{n}+a_{n} t}-S_{n-a_{n}}\right\}, \quad t=i / a_{n}, 0<i<a_{n}, \\
& =\text { linear for other } t, \quad 0 \leqslant t \leqslant 1 .
\end{aligned}
$$

The main result of this note is

THEOREM 1. Under condition (1), with probability one, the sequence of random elements $\left(g_{n}\right)$ in $C_{H}[0,1]$ is relatively compact and has $U_{\mu}$ as its set of limit points.

We will only sketch the proof of this theorem. First some lemmas.

LEMMA 1. Let $\left(\mu_{n}\right)$ be a sequence of zero-mean Gaussian measures on $H$ with associated covariance operators $T_{n}$. If $T_{n}$, s converge in the trace-norm topology to an operator $T$ then $\mu_{n}$ 's converge weakly to a zero-mean Gaussian measure $\mu$ on $H$ whose covariance operator is $T$.

Proof. This is probably well known. In any case it follows easily from Lemma 5.1 on p. 182 of Parthasarathy [13].

LEMMA 2. Let $\left(X_{n}, Y_{n}\right)$ have joint zero-mean Gaussian distribution on $H \times H$ with marginal covariance operators $T_{n}, T_{n}^{\prime}$ respectively and cross-covariance operator $K_{n}$. Suppose in the trace-norm topology we have $T_{n} \rightarrow T, T_{n}^{\prime} \rightarrow T^{\prime}$ and $K_{n} \rightarrow 0$ (i.e. the trace norm of $K_{n}$ goes to zero). Then the distribution of $\left(X_{n}, Y_{n}\right)$ converges weakly to the product measure $\nu \times \nu^{\prime}$ where $\nu, \nu^{\prime}$ are the zero-mean Gaussian measures on $H$ with the covariance operators $T, T^{\prime}$ respectively.

Proof. Straightforward. 
LEMMA 3. Let $Z$ have a zero-mean Gaussian distribution on $H$ with covariance operator $T$. Then for all $\lambda>0$,

$$
E[\exp (\lambda\|Z\|)]<8 \exp \left(16 \sigma^{2} \lambda^{2}\right)
$$

where $\sigma^{2}$ is the trace-norm of $T$.

Proof. Let $\left(\alpha_{n}\right)$ be the eigenvalues of $T$. Then $\Sigma_{n} \alpha_{n}=\sigma^{2}$ and $Z$ can be written as $Z=\Sigma_{n} \xi_{n} e_{n} \sqrt{\alpha_{n}}$ where $\xi_{n}$ 's are real-valued, independent $N(0,1)$ r.v's and $\left(e_{n}\right)$ is an orthonormal collection in $H$. Hence

$$
E\left[\exp \left(s\|Z\|^{2}\right)\right]=\prod_{n}\left(1-2 \alpha_{n} s\right)^{-1 / 2},
$$

for all $s$ less than $(2 x \text { maximal eigenvalue of } T)^{-1}$. Now let $s_{0}=\left(8 \sigma^{2}\right)^{-1}$. Using the elementary inequality $(1-u)^{-1 / 2} \leqslant 1+u \leqslant e^{u}, \forall u \in(0,1 / 2)$ we see that $E\left[\exp \left(s_{0}\|Z\|^{2}\right)\right]<\exp \left(2 s_{0} \Sigma_{n} \alpha_{n}\right)=e^{1 / 4}$. Hence $P[\|Z\|>u]<e^{1 / 4} \cdot e^{-s_{0} u^{2}}, \forall u>0$ and thus

$$
\begin{aligned}
E[\exp (\lambda\|Z\|)] & =\int_{0}^{\infty} \lambda e^{\lambda u} P[\|Z\|>u] d u \\
& \leqslant e^{1 / 4} \int_{0}^{\infty} \lambda e^{\lambda u-s_{0} u^{2}} d u \\
& =e^{1 / 4} \int_{0}^{\infty} \lambda e^{-s_{0}}\left(u-\lambda / 2 s_{0}\right)^{2} \cdot e^{\lambda^{2} / 4 s_{0}} d u \\
& \leqslant e^{1 / 4} \cdot e^{\lambda^{2} / 4 s_{0}} \cdot \lambda \int_{-\infty}^{\infty} e^{-s_{0}\left(u-\lambda / 2 s_{0}\right)^{2}} d u \\
& =e^{1 / 4} \cdot \sqrt{2 \pi} \sqrt{2}\left(\lambda / \sqrt{4 s_{0}}\right) \exp \left(\lambda^{2} / 4 s_{0}\right) .
\end{aligned}
$$

Considering separately the cases when $\lambda / \sqrt{4 s_{0}}$ is $<1$ or $>1$ and substituting $s_{0}=\left(8 \sigma^{2}\right)^{-1}$ the assertion of the lemma is easily seen to follow.

LEMMA 4. Under the conditions of Theorem $1, \exists A>0$ such that, for all $n$ and all $\lambda>0$, we have

$$
E\left[\exp \left(\lambda \max _{1<j<n}\left\|S_{j}\right\|\right)\right]<64 \exp \left(A \lambda^{2} n\right)
$$

Proof. We have already seen that the covariance operators of $S_{n} / \sqrt{n}$ converge in the trace-norm topology and therefore have their trace-norms bounded by a finite constant, say $B$. We now apply the preceding lemma and the elegant Theorem 1 of Móricz [12] and see that we can take $A$ to be $16 \times 12 B$. Note that although Móricz [12] deals only with real-valued sequences his Theorem 1 extends directly to our set-up since only the triangle inequality is involved.

Returning now to the proof of Theorem 1 let us first show that the sequence of random processes $\left(g_{n} \sqrt{2 b_{n}}\right)$ converges weakly in $C_{H}[0,1]$ to the $\mu$-Wiener process. For this we need to verify convergence of finite-dimensional distributions and tightness. Convergence of one-dimensional distributions follows from Lemma 1. Asymptotic independence of increments over strictly separated intervals is easily seen to follow from Lemma 2. The usual moment condition for tightness extends directly to the set-up here as is shown in Theorem 2, p. 410 of Gihman and 
Skorohod [8]. This theorem applies here with $\alpha=4$ and $\beta=2$, thereby establishing tightness.

To prove the upper and lower class results in Theorem 1 we introduce two sequences of integers. Let $\varepsilon>0$. Define $n_{k}=n_{k}(\varepsilon)$ and $m_{k}=m_{k}(\varepsilon)$ by

$$
\begin{aligned}
n_{k} & =\left[(1+\varepsilon)^{k}\right] \quad \text { if } \lim _{n}\left(a_{n} / n\right)>0, \\
& =\min \left\{m: m \log m / a_{m} \geqslant k \varepsilon\right\} \quad \text { if } \lim _{n}\left(a_{n} / n\right)=0 .
\end{aligned}
$$

Also, $m_{k}=n_{k}$ if $\lim _{n}\left(a_{n} / n\right)>0$, and

$$
m_{k}=\min \left\{m: m \log m / a_{m} \geqslant k^{1+e}\right\} \quad \text { if } \lim \left(a_{n} / n\right)=0 .
$$

Because of the assumed conditions on the sequence $\left(a_{n}\right)$ it is clear that these sequences exist and increase to infinity. Let us now verify

$$
b_{n_{k}} \sim \log k
$$

and

$$
\begin{aligned}
n_{k+1}-n_{k} & \leqslant 2 \varepsilon c^{-1} a_{n_{k}} \quad \text { for large } k \text { if } \lim _{n}\left(a_{n} / n\right)=c>0, \\
& \leqslant 2 \varepsilon a_{n_{k}} \text { for large } k \text { if } \lim _{n}\left(a_{n} / n\right)=0 .
\end{aligned}
$$

In the case $\lim _{n}\left(a_{n} / n\right)=c>0,(2)$ is trivial and (3) follows because $a_{n}>n c$.

Now let $\lim _{n}\left(a_{n} / n\right)=0$. In this case we first verify

$$
c_{n+1}-c_{n} \geqslant 1 / a_{n+1} \quad \text { where } c_{n}=n \log n / a_{n} .
$$

Indeed define the function $a(x)$ on the interval $[n, n+1]$ by $a(n)=a_{n}, a(n+1)=$ $a_{n+1}$ and linear in between. Then $a(x)$ is increasing on $[n, n+1]$ and it is trivial to check that the condition $a_{n+1} / a_{n} \leqslant(n+1) / n$ implies that the function $f(x)=$ $x / a(x)$ is also increasing on $[n, n+1]$. Now the derivative of $f(x) \log x$ is at least $f(x) / x$ since $f^{\prime}(x) \geqslant 0$. Hence by the mean-value theorem,

$$
\begin{aligned}
c_{n+1}-c_{n} & \geqslant f\left(x^{*}\right) / x^{*} \text { for some } x^{*} \in[n, n+1) \\
& =1 / a\left(x^{*}\right) \geqslant 1 / a_{n+1} .
\end{aligned}
$$

This is (4). Now to prove (2) and (3) in this case we have $1<a_{n+1} / a_{n}<(n+1) / n$ implies $a_{n+1} \sim a_{n}$ and so $c_{n+1} \sim c_{n}$. Thus $c_{n_{k}}-1 \leqslant k \varepsilon<c_{n_{k}}$ implies $c_{n_{k}} \sim k \varepsilon$ and hence $b_{n_{k}}=\log c_{n_{k}} \sim \log k$. This proves (2). Also

$$
\begin{aligned}
\varepsilon & =(k+1) \varepsilon-k \varepsilon \geqslant c_{n_{k+1}}-c_{n_{k}} \\
& \geqslant\left(n_{k+1}-n_{k}-1\right)\left(1 / a_{n_{k+1}}\right)
\end{aligned}
$$

by (4) and because $a_{n} \uparrow$. Hence $n_{k+1}-n_{k}-1 \leqslant \varepsilon a_{n_{k+1}}$. Dividing through by $n_{k+1}$ and noting that $a_{n} / n \downarrow 0$ we get $n_{k+1} \sim n_{k}$ and since $a_{n} / n \downarrow$ we find $a_{n_{k+1}} \sim a_{n_{k}}$. Hence $n_{k+1}-n_{k} \leqslant 1+\varepsilon a_{n_{k+1}} \leqslant 2 \varepsilon a_{n_{k}}$ for all large $k$. Thus (2) and (3) are completely proved.

Let us now show that the sequence $\left(g_{n}\right)$ is relatively compact in $C_{H}[0,1]$ and in fact $d\left(g_{n}, U_{\mu}\right) \rightarrow 0$ a.s. where $d$ is the usual distance of a point from a set. For this purpose we want to apply Theorem 4.1 of Carmona and Kôno [2] to the sequence $Y_{k}=g_{n_{k}} \sqrt{2 b_{n_{k}}}$. We have already seen that $\left(Y_{k}\right)$ converges to the distribution of the $\mu$-Wiener process. In the case $a_{n} / n \downarrow 0$, the subsequence $n_{k}$, however, may not be 
sparse enough to yield the condition (4.2) of [2]. However notice that even if one drops the condition (4.2) in Theorem 4.1, compactness of $\left((2 \log k)^{-1 / 2} Y_{k}\right)$ is established without using (4.2). Given the relative compactness one can apply part I of Theorem 3.1 of Kuelbs [9] since the condition (3.1) of [9] is automatically satisfied here because of the convergence of the Gaussian sequence $\left(Y_{k}\right)$. Thus we see that $d\left(g_{n_{k}}, U_{\mu}\right) \rightarrow 0$ a.s. Now as shown e.g. in equations (2.6), (2.7) of [12] the bound on the moment generating function in Lemma 4 yields a corresponding bound on the tail of the distribution. Using this and (3) we easily conclude that given $\delta>0$ we can find $\varepsilon>0$ such that (with $n_{k}=n_{k}(\varepsilon)$ ) $\max _{n_{k}<n<n_{k+1}}\left\|g_{n}-g_{n_{k}}\right\|$ exceeds $\delta$ only finitely often with probability one. Thus $d\left(g_{n}, U_{\mu}\right) \rightarrow 0$ a.s. completing the upper-half part of the proof.

For the lower-half part of the proof we use the subsequence $m_{k}=m_{k}(\varepsilon)$. We show that Theorem 4.1 of Carmona and Kôno [2] applies to the sequence $Y_{k}=g_{m_{k}} \sqrt{2 b_{m_{k}}}$. We have only to check the condition (4.2). For this we need to know the form of the elements in the dual of $C_{H}[0,1]$. If $f \in C_{H}^{*}[0,1]$ there exists a bounded mapping $G:[0,1] \rightarrow H$ and a finite positive Borel measure $\gamma$ on $[0,1]$ such that

$$
f(x)=\int_{0}^{1}\langle G(t), x(t)\rangle d \gamma(t) \quad \forall x \in C_{H}[0,1] .
$$

This is given e.g. in Theorem 5, p. 389 of Dinculeanu [6]. It follows from this that

$$
E\left\{\left\langle f, Y_{l}\right\rangle\left\langle f, Y_{l^{\prime}}\right\rangle\right\}=\int_{0}^{1} \int_{0}^{1}\left\langle K_{l, l^{\prime}}(s, t) G(s), G(t)\right\rangle d \gamma(s) d \gamma(t)
$$

where we have written $K_{l, l^{\prime}}(s, t)$ for the cross-covariance operator between $Y_{l}(s)$ and $Y_{l^{\prime}}(t)$. Thus to verify the condition (4.2) of [2] it certainly suffices to show that

$$
\limsup _{\substack{l \rightarrow \infty \\ l \rightarrow l}} \sup _{0<s, t<1}\left\{\text { trace norm of } K_{l, l^{\prime}}(s, t)\right\}=0 .
$$

Consider first the case $\lim _{n}\left(a_{n} / n\right)=0$. In this case it follows from our definition of $m_{k}$ that $\left(m_{k+1}-a_{m_{k+1}}\right)-m_{k} \geqslant k^{\varepsilon} \geqslant 1$. Hence using the arguments leading to (6) in Deo [5] it is clear that (under our condition (1)) with $l<l^{\prime}$

$$
\sup _{0<s, t<1}\left\{\text { trace norm } K_{l, l^{\prime}}(s, t)\right\}=O\left(a_{m_{l}}^{-\beta}\right) .
$$

Hence (5) follows. In the case $\lim _{n}\left(a_{n} / n\right)>0$ the arguments leading to (6) and (7) in [5] show that the left-hand expression in (6) above is dominated by a constant multiple of $\left[(1+\varepsilon)^{\left(l-l^{\prime}\right) / 2}+(1+\varepsilon)^{-l \beta}\right]$. Again (5) above follows. Thus Theorem 4.1 of [2] applies to $Y_{k}$. Note that if $\lim _{n}\left(a_{n} / n\right)>0$ then $b_{m_{k}} \sim \log k$ and if $\lim _{n}\left(a_{n} / n\right)=0$ then $b_{m_{k}} \sim(1+\varepsilon) \log k$. Thus in the former case we get the cluster of $\left(g_{n}\right)$ contains $U_{\mu}$ a.s. and in the latter case that the cluster set of $\left(g_{n}\right)$ contains $(1+\varepsilon)^{-1 / 2} U_{\mu}$ a.s But since $\varepsilon>0$ is arbitrary the proof of Theorem 1 is completed.

Let $B$ be a separable Banach space and $\mu$ a zero-mean Gaussian measure on $B$ and $\{W(t): 0 \leqslant t<\infty\}$ a $\mu$-Wiener process. Let $U_{\mu}$ be the unit ball in the RKHS of $\{W(t): 0 \leqslant t \leqslant 1\} . U_{\mu}$ is a compact subset of $C_{B}[0,1]$. Let the constants $a_{n}, b_{n}$ 
be as before and write $g_{n}(t)=\left(2 a_{n} b_{n}\right)^{-1 / 2}\left\{W\left(n-a_{n}+a_{n} t\right)-W\left(n-a_{n}\right)\right\}, 0<t$ $<1$. Then the method of proof employed for Theorem 1 easily yields

THEOREM 2. In the above framework, with probability one, the sequence $\left(g_{n}\right)$ is relatively compact in $C_{B}[0,1]$ and has $U_{\mu}$ as its set of limit points.

Note that Theorem 2 extends the results in [3], [10] and [11].

3. Concluding remarks. It would be possible to obtain an analog of Theorem 1 for $B$-valued, stationary Gaussian sequences. However because of the absence of a nice sufficient condition (cf. Lemma 1) for convergence of Gaussian measures on $B$ it is unlikely that such a theorem will have a neat form.

It should be noted that even in the case $H=R$, our Theorem 1 cannot be obtained from the theorem in [3] and the a.s. invariance principle for Gaussian sequences in [14]. The order of approximation in the latter is not fine enough.

Acknowledgement. I am indebted to Professors M. Csörgö and P. Révész and to Dr. A. H. C. Chan for introducing me to this area by communicating their results to me. I also wish to thank the referee for an excellent job.

\section{REFERENCES}

1. C. R. Baker, Joint measures and cross-covariance operators, Trans. Amer. Math. Soc. 186 (1973), 273-289.

2. R. Carmona and N. Kôno, Convergence en loi du logarithme iteré pour les vecteurs gaussiens, $\mathbf{Z}$. Wahrscheinlichkeitstheorie und Verw. Gebiete 36 (1976), 241-267.

3. A. H. C. Chan, M. Csörgö and P. Révész, Strassen type limit points for moving averages of a Wiener process, Canad. J. Statist. 6 (1978), 57-75.

4. M. Csörgö and P. Révész, How big are the increments of a Wiener process, Ann. Probability 7 (1979), 731-737.

5. C. M. Deo, A note on stationary Gaussian sequences, Ann. Probability 2 (1974), 954-957.

6. N. Dinculeanu. Vector measures, Pergamon Press, Berlin and New York, 1967.

7. I. M. Gelfand and N. Ya. Vilenkin, Generalized functions, vol. 4, Academic Press, New York, 1964.

8. I. I. Gihman and A. V. Skorohod, The theory of stochastic processes. I, Springer-Verlag, Berlin and New York, 1974.

9. J. Kuelbs, A strong convergence theorem for Banach space valued random variables, Ann. Probability 4 (1976), 744-771.

10. J. Kuelbs and R. Lepage, The law of the iterated logarithm for Brownian motion in a Banach space, Trans. Amer. Math. Soc 185 (1973), 253-264.

11. T. L. Lai, On Strassen-type laws of the iterated logarithm for delayed averages of the Wiener process, Bull. Inst. Math. Acad. Sinica 1 (1973), 29-39.

12. F. Moricz, Probability inequalities of exponential type and laws of the iterated logarithm, Acta Sci. Math. 38 (1976), 325-341.

13. K. R. Parthasarathy, Probability measures on metric spaces, Academic Press, New York, 1967.

14. W. Philipp and W. Stout, Almost sure invariance principles for partial sums of weakly dependent random variables, Mem. Amer. Math. Soc., No. 161 (1975).

Department of Mathematics, Universtty of Ottawa, Ottawa, Ontario K1N 9B4, Canada 